\title{
The Role of Science Centres as Aids for Astronomical Education
}

\author{
By J. Fierro \\ Instituto de Astronomia, UNAM, Mexico
}

\section{Introduction}

Education is training, part of which is being able to handle information. At meetings such as this, one learns new ways to teach and to adapt ideas to one's culture in a way they can have a greater influence on the lay person (Pasachoff and Percy, 1990; Percy, 1996). A reason to promote science popularization is to give people a chance to experience the pleasure of understanding.

Traditionally written materials and planetariums were the ideal way to convey astronomical knowledge and to start an interest in science. Now the media, WWW and interactive exhibits are having a great influence on the lay person. Science centres are an important aid for education; they present astronomy in an attractive way, which is sometimes difficult to do at school. It is easier to teach something that pupils enjoy.

This paper will focus on science centres in Mexico; some of the ideas that we have used could help other developing nations with their projects. In order to grasp the differences between other countries and Mexico, I shall only mention that the average education is five years in large cities nad two in the country; 78 million, out of 95 million inhabitants, never buy a book, and only 1 million purchase more than 10 books per year; the introductory astronomy course that is taught to over 200,000 students per year in the USA is only taught to about 60 pupils per year at our National University.

We shall describe some of the activities that science centres can provide in order to aid public understanding of astronomy and the ways in which several very small museums have been installed in Mexico.

\section{Science Centres}

Science centres throughout the world have incorporated many of the resources used for public education: hands-on activities, formal exhibits (including texts, pictures and models), computers, workshops, theatre, publications and videos. Since every person is different, the more varied objects an exhibit presented, the more likely it will succeed in reaching a broader public.

Science centres have to adapt continuously to different audiences. For instance, in the USA elderly people are becoming an important part of the visitors, so labels have to have large type and provide interesting information for such a group (sometimes they may also have to include bilingual information).

I think every major city should have a good science centre with strong outreach programs, whereas small communities should focus more on small facilties, where emphasis is on workshops, teaching resources, and travelling exhibits, including major astronomical events such as comets or solar eclipses.

Every science centre should have ties with universities and draw from its local culture, otherwise it is very difficult for it to come up with new ideas and have a strong influence on the general public.

Mexico city has a large science centre: Universum, where two halls are dedicated to astronomy, with sixty interactive exhibits aimed at several age levels. Now a colonial 
ex-convent will house a science center whose theme will be electromagnetic radiation; it will also have an exhibit on the use of spectroscopy for astronomical purposes in its ex-chapel which has a domed ceiling. (Several displays have been tried out at book fairs where their designers receive feedback from the public.)

Universum has fostered displays in places as diverse as the Palace of Fine Arts accompanied by a performance of the National Symphonia Orchestra playing astronomical works, to lobbies of subway stations where people who would have difficulty going to a museum get a chance to see state of the art science exhibits (Fierro, 1993, 1994, 1995).

Universum has produced several low cost plays, with astronomical topics, that can easily travel to other communities; the latest is on evolution; it uses a darkened stage where actors, using flourescent garments and puppets, stage spiral galaxies, the solar system and the evolution from fish to frog, while a narrator describes main cosmological events.

\subsection{Hands-on exhibits}

Astronomical exhibits are some of the most popular, only comparable to the ones on our body and dinosaurs. Hands-on exhibits have proven extremely successful since there is direct correlation between the amount of activity and the quantity of information one remembers. They should appeal to all senses; for instance in Mexico we have exhibits on the way different worlds would smell, sound and feel if we could visit them.

There are splendid astronomical displays all over the world, many of which are adjacent to planetariums. I shall only mention a few example out of the hundreds of interesting displays that have been constructed all over the world that aid astronomy teaching. For instance, at the Deutsches Museum, the spectroscopy exhibit features a historical approach, challenging information, sturdy manipulating devices and topics for all age levels. As an example, it includes a sort of slide rule where the Doppler shift of a galaxy and a quasar can be measured by comparing certain absorption lines. In Spain there is an exhibit where the visitor has to elaborate a virtual message using a computer and has to convey it via a radio signal to some sort of unknown extraterrestrial being. This sort of activity motivates creativity and helps people understand the difficulty of trying to communicate with extraterrestrial intelligence. In Mexico, the display on seasons, first focuses on extreme conditions: Jupiter and Uranus, and then on intermediate ones: Earth and Mars. La Villete in France offeres materials for teachers and long term activities for school children.

Extreme care must be given when designing exhibits; the Ontario Science Centre has managed to create hundreds of interactive exhibits trying them out with visitors in a preliminary version, before constructing the final item.

In the developing countries hands-on apparatus are crucial in order to give students a chance to experiment. Many schoold have no laboratories and middle school teachers usually tend to give lectures.

\subsection{The Travelling Museum}

The Ontario Science Centre has a long tradition in travelling exhibits that have proven to be a most successful way of conveying scientific information and hands-on experiences to small communities. They can be placed at book fairs, cultural centres, schools and malls.

We have found that one of the most convenient places to place displays are large lobbies at subway stations; they can include simple workshops and public lectures. Hundreds of thousands of people use the subway and during school hours children can visit the exhibits at low cost (in Mexico the ticket for the underground is $\$ 0.15$ U.S.). 
Currently an exhibit on "the way Olympic Games would be held in other worlds of the solar system" is under design, sponsored by a sports-related National Lottery.

\subsection{Workshops}

Workshops are extremely important for education because students can create something manually. Apart from being good for conveying information and for the visitors' psychomotor development, students learn how to build objects: robots, models of extraterrestrial creatures, telescopes, sundials, three dimensional constellations or rockets, (Fraknoi, 1995; Jackson, 1994; VanCleave, 1991; Walusinski, 1989). Concluding a task is something education in Mexico strongly needs because many people have great projects but have difficulty turning them into reality.

\subsection{Public Lectures}

When science centres have strong links with universities they can provide high quality public lectures and panel discussions for a large variety of audiences if they use video conference facilties.

Listening carefully for the questions people address can be valuable to learn about their misconceptions and interests. One of the most successful books I have written is based on the answers to questions raised during my public lectures.

\subsection{Ushers}

Some of the science centres train ushers; they are usually undergraduate students. Many of them have such pleasant experiences at the centres that they later pursue scientific careers. Ushers can become the role models for small children. Amateur astronomers can be extremely good ushers specially for outreach programs.

\subsection{Plays}

Plays are attractive ways to convey scientific knowledge. Some of the scripts are simple enough so teachers can later encourage pupils to interpret their own plays. (One of the middle school geography books encourages students to make a play on the properties of the solar system.)

\subsection{Libraries}

Several science centres are equipped with libraries which hold collections that teachers and pupils can use; these may be the only existing specialized libraries. Several hold videos and private sections for small children. Science centres are producing audiovisual material that can be purchased by teachers. Some museums have visitor-oriented computers with on-line facilities where teachers can find information necessary to update their courses. Unfortunately most information on the WWW is in English, so developing nations must make an effort to include more material and point out the necessity of teaching a second language at school.

Most major museums and science centres have home pages on the WWW. The information varies greatly, including historical backgrounds, virtual visits to the major exhibits; a few include diagrams of interactive displays.

\subsection{Shops}

Science centres tend to have shops where instructors can purchase materials: science kits, information posters, videos, computer programs, books and magazines where they can find activities they can carry out before and after the visit, as well as workshops and updated information. In many cases such stores are the only places to buy educational items. 


\subsection{Teaching materials}

Several science centres produce teaching materials. For instance the San Francisco $E x-$ ploratorium and the Ontario Science Center have a series of books on experiments that do not require complicated equipment. Some edit newsletters, electronic and not, that include science information that can be very useful for elementary school teachers, especially in the case where local newspapers do not print science columns.

In Mexico a group of teachers edited a new magazine and asked scientists that collaborated with Universum to write small articles for them, including hands-on activities. Some of the astronomy articles have been inspired by the work done by the French group published in the Cahiers Clairaut and other CLEA publications (Walusinski, 1989-1996).

\subsection{Starlabs and small telescopes}

Starlabs, portable planetariums, are extemely useful teaching laboratories. They can be adapted to meet the requirements for every school level and should be included in the outreach programs of science centres. They can also adapt telescope viewing with their presentations.

In many schools in large cities a visit to a planetarium is compulsory, so it is important that they provide high quality shows. Here professional astronomers can provide invaluable aid if they referee the scripts.

\subsection{Amateurs}

Several science centres have planetariums and small telescopes; some shows can be conducted by amateurs, especially since they tend to be an enthusiastic group. They can contribue to outreach activities such as star parties and Astronomical Extravanganza (camping inside the dome). One must make sure that the amateurs involved not only convey enthusiasm but also have a sense of scientific knowledge.

Science centers can promote the creation of amateur groups especially if they have a planetarium or a telescope.

\section{Very small science centers}

\subsection{Science Wagons}

One of the most successful projects of promotion of science in Mexico has been called $A$ Wagon for Science. It consists of discontinued train coaches, and more recently, trucks and airplanes, remodeled to hold laboratory facilities and libraries. Some of them are associated with theatres where public lectures, promoted by Mexico's National Academcy of Sciences, are given on Sunday mornings .

\subsection{Starting a Science Center}

Major cities in developing nations should have large science centers similar to those constructed in developed nations, and towns more workshop oriented facilities. Seven large and twenty-three small centers have been installed in Mexico; I shall outline some of their characteristics. One must be very careful when planning a new center: a museum at Aguascalientes is devoted to evolution; unfortunately its exhibits are rigid and difficult to update; it only serves 450,000 people and has no feedback by a scientific staff. A children's museum in Hermosillo is an almost exact copy of one in Mexico City, including the same mistakes on its labels!

An ideal way to start a new science center in a developing country is to purchase a 
Starlab, a small telescope, install a Science Wagon and construct a few simple astronomical hands-on exhibits. These items should be tried out in small communities in order to get experience and obtain local support.

Developing nations must have as many interactive, travelling, exhibits as possible, not only because they have proven to be good for conveying scientific knowledge, but also because in many cases they are the only laboratories students will ever visit. It is necessary to have areas dedicated to workshops and other continually changing activities, otherwise the public will stop visiting and the museum will deteriorate rapidly from the lack of visitors. New science centers should use the vast experience developed all over the world but should use local skills.

All projects should have a strong link with teachers. They should provide follow up materials. Teachers, particularly in developing nations, need basic information that will help them cope with children's questions.

A good way to start getting a community's enthusiasm for a new science center is a "Market of Science" or a "Scientific Caravan". The market of science is a science fair where several interactive experiments and workshops are presented to the public. The scientific caravan is a museum on wheels where the fair travels to a large number of small communities. This kind of activity provides a good way to try out exhibits. Two universities in small cities in Mexico have begun this kind of activity with good results at Morelia and Sonora.

The final and most important suggestion is that any science center project must have strong links with local universities. This is fundamental in order to ensure conveying proper information and, especially, to constantly renew the exhibits.

\section{Conclusion}

Science centers can provide a wide range of aids for astronomical education. When new small science centers are opened in developing nations they should emphasize activities devoted to outreach programs, such as travelling exhibitis, workshops, teacher assistance, and promote self education. They should be associated with universities and draw from local cultures.

Even though science centers provide exciting expreiences they cannot substitute formal education; astronomers should collaborate with them in order to convey to a large population the pleasure of understanding.

\section{REFERENCES}

Fierro, J. \& Doddoli, C., Museum News, May-June 1993, p.35.

Fierro, J., Asian-Pacific Teaching of Astronomy, Vol. 8, Ed. S. Isobe, p1-15.

Fierro, J.,, Highlights of Astronomy, 10, Kluwer Academic Publishers.

FraKnol, A. Ed., 1995, Project ASTRO, Astronomical Society of the Pacific.

Jackson, S., Ed., 1994, How the Universe Works, Reader's Digest Association.

Pasachoff, J.M. \& Percy, J.R., Ed., 1990, The Teaching of Astronomy, Cambridge, Cambridge University Press, IAU Colloguium no. 105.

Percy, J., Ed., 1996, Astronomy Education: Current Developments, Future Coordination, Astronomical Society of the Pacific Conference Series, Vol. 89.

VanCleave, J., Astronomy for Every Kid, J. Wiley.

WALUsinski, G., Ed., 1989-1996, Les Cahiers Clairaut, CLEA. 